\title{
Los recursos educativos digitales en la atención a la diversidad en Educación Infantil
}

\author{
Digital educational resources in attention \\ to diversity in Early Childhood Education
}

RECIBIDO 27/3/2021 ACEPTADO 8/6/2021 PUBLICADO 1/12/2021

\author{
(D) María López Marí \\ Grupo de Investigación CRIE (GIUV2013-105), Universidad de Valencia, España \\ maria.loma4@gmail.com \\ (D) Melanie Sánchez Cruz \\ Grupo de Investigación CRIE (GIUV2013-105), Universidad de Valencia, España \\ mesan4@alumni.uv.es \\ José Peirats Chacón \\ Departamento de Didáctica y Organización Escolar, Universidad de Valencia, España \\ Jose.Peirats@uv.es
}

\section{RESUMEN}

La etapa de Educación Infantil asume un papel fundamental en la prevención e intervención temprana de las necesidades educativas que puedan presentar los niños y niñas durante su desarrollo. Para el tratamiento de estas individualidades, que pueden ser permanentes o transitorias, los recursos educativos digitales se han convertido en eficaces herramientas de apoyo gracias a su carácter motivador y personalizable. Esta transformación digital de los materiales didácticos, que continúan combinándose con los tradicionales y manipulativos, han dado lugar a cambios metodológicos y organizativos complejos. De todos ellos, en este estudio nos proponemos analizar en qué medida estos recursos facilitan el tratamiento de la diversidad en el segundo ciclo de Educación Infantil (3 a 6 años) y permiten la realización de prácticas escolares más inclusivas. Para ello, se examinarán las características técnicas y pedagógicas de diversos materiales proporcionados por editoriales e instituciones dirigidos al alumnado de infantil. Los resultados indican que son materiales interactivos con retroalimentación inmediata a los participantes. Cuentan con interfaces y narrativas atractivas para niños y niñas de esa etapa, con efectos visuales, sonoros. Y, finalmente, favorecen un aprendizaje multisensorial con uso de distintos canales de información.

PALABRAS CLAVE educación infantil, recursos educativos, innovación educativa, diversidad del alumnado, inclusión educativa.

\section{ABSTRACT}

The Early Childhood Education stage assumes a fundamental role in the prevention and early intervention of the educational needs that children may have during their development. For the treatment of these individuals, which can be permanent or transient, digital educational resources have become effective support tools thanks to their motivating and customizable character. This digital transformation of teaching materials, which continue to be combined with traditional and manipulative materials, has led to complex methodological and organizational changes. Of all of them, in this study we propose 
to analyze to what extent these resources facilitate the treatment of diversity in the second cycle of Early Childhood Education ( 3 to 6 years) and allow the realization of more inclusive school practices. This will examine the technical and pedagogical characteristics of various materials provided by publishers and institutions aimed at children's students. The results indicate that they are interactive materials with immediate feedback to the participants. They have attractive interfaces and narratives for boys and girls of that stage, with visual and sound effects. And, finally, they favor multisensory learning with the use of different information channels.

KEYWORDS early childhood education, educational resources, educational innovation, student diversity, inclusive education.

\section{INTRODUCCIÓN}

La omnipresencia de las tecnologías en nuestra sociedad y los cambios socioculturales que ha impulsado son notables. Para plasmar este fenómeno acaecido en los últimos años, Area y Pessoa (2012) emplean la metáfora de Bauman, destacando que la cultura digital ha derivado en la producción de información continuada y en una formación y conocimiento en constante transformación, frente a la inmutabilidad de lo material propia de los siglos XIX y XX. Esto se puede extrapolar a aquello que sucede en las escuelas donde, con anterioridad a la entrada de las Tecnologías de la Información y la Comunicación (TIC), ya se cuestionaba el papel desempeñado por los libros de texto. Sin embargo, pese al gran crecimiento de las TIC, en uso y presencia, quienes actualmente siguen teniendo un papel principal en las aulas son los libros de texto (Rodríguez, \& Rodríguez, 2016, San Martín, \& Peirats, 2018). Aunque no se vislumbra cuando cambiará el sentido de la balanza, es indudable que la colonización de lo digital en el mundo editorial se ha extendido de forma muy evidente hacia el proceso de elaboración de materiales didácticos digitales (MDD) (Sanabria et al., 2017; Santana et al., 2017).

La definición y sistematización de los distintos MDD es considerablemente compleja. Quien se ha ocupado es Area (2017), al elaborar un inventario que engloba los recursos educativos digitales en su conjunto, en función de los materiales encontrados en diversos portales educativos, institucionales y comerciales. No entraremos a enunciarlos, pero sí se señala que diferencia en su clasificación entre objeto digital, de aprendizaje, entorno didáctico digital, libro de texto digital, apps, herramientas y plataformas online, entornos inteligentes de aprendizaje, materiales didácticos tangibles y materiales digitales para la docencia.

Entre todos ellos, como ejemplo de su pujanza, el sector de las App se encuentra en pleno proceso de crecimiento (únicamente en Apple Store podemos encontrar más de 80.000 aplicaciones), lo que puede interpretarse como un interés creciente por parte de las familias y el profesorado en introducir este tipo de materiales tanto en las aulas como en la escuela (Troseth et al., 2016). No obstante, no todo deslumbra en este proceso y se encuentra que la gran mayoría de recursos etiquetados como "educativos", no han sido todavía correctamente evaluados y se observan déficits tanto a nivel de contenido como de diseño (Rodríguez et al., 2015).

Desde el ámbito educativo, son múltiples las aportaciones de la investigación realizada en la última década que permiten la evaluación de los recursos educativos digitales. Entre ellos, se señala la de Fernández et al. (2011), donde contemplan diez dimensiones: documentación didáctica; calidad de los contenidos; reflexión, crítica e innovación; interactividad y adaptabilidad; motivación; formato y diseño; usabilidad; ac- 
cesibilidad; reusabilidad; e interoperabilidad. Mientras, Aguilar et al. (2014) contemplan la dimensión pedagógica y tecnológica y atienden a dos niveles de análisis, el de diseño y el de uso, y señalan como principales criterios de evaluación los siguientes aspectos: considerar el contexto de uso de los MDD, atendiendo a las estrategias de enseñanza, características del alumnado, el nivel de interactividad del material o como está presentado; la creación y evaluación debe estar desarrollada por profesionales de diversos perfiles; y la evaluación de los materiales se ha de llevar a cabo durante diversas etapas, tanto cuando se desarrolla como después de su uso, atendiendo a dimensiones o componentes. La coordinada por Peirats et al. (2016), estructurada en torno a tres grandes criterios: el pedagógico, el tecnológico y el económico. O, más recientemente, la presentada por los grupos de investigación EDULLAB, CRIE y STELLAE (2019) que, en formato de guía, orienta sobre la producción y uso de los MDD. Sin entrar en profundizar o revisar más clasificaciones, se destaca que la calidad en el tema que nos ocupa reside, principalmente, en que el material didáctico empleado, sea digital o no, se adapte al momento evolutivo del niño o niña y sea accesible y flexible para ofrecer una mejor atención a la diversidad del alumnado del aula (Gabarda et al., 2021).

Los beneficios de los recursos digitales son múltiples, nadie lo duda; por un lado, se ha observado que los juegos digitales provocan una mejora en las habilidades sociales, especialmente en constructos como el autoconcepto, la autoeficacia o el reconocimiento de emociones propias, así como una optimización en las habilidades sociales y comunicativas de personas con autismo (Durán, 2021) y síndrome de Down (Porter, 2018). Asimismo, promueven una mejora en los procesos de motivación, creatividad e imaginación, en la resolución de problemas y un mayor desarrollo de la autonomía y organización del propio aprendizaje. También pueden emplearse para trabajar de manera colaborativa y permite adaptarse a las necesidades y características intrínsecas del alumnado, mediante la realización de pautas, programas o adaptaciones individualizadas (Peirats et al., 2015), generando así un aprendizaje activo que fomenta la competencia de aprender haciendo, frente al modelo tradicional de comunicación unidireccional. Por último, permiten trabajar a distintos niveles, adecuándose a los diversos estilos, momentos o espacios de aprendizaje (Fombona, \& Vázquez-Cano, 2017), aspecto especialmente relevante en el ámbito tratado. Cabe reseñar, sin embargo, que estas características favorecedoras no conllevan u obligan a que se dejen de lado ciertos mecanismos de control o monitorización continua, y que en este caso son menos perceptibles (Page, 2016).

Pese a todo lo enunciado, también se encuentran sombras en el camino, ciertas dificultades o problemas relacionados con la conocida por todos como brecha digital y sus consecuencias. Burbules (2012) nos advierte acertadamente sobre el carácter y naturaleza de las mismas:

\footnotetext{
No sólo se trata de una "brecha digital"; es una división de todo tipo de otras oportunidades. Chances desiguales para aprender a aprovechar el acceso a la tecnología, posiblemente; desigualdad en las redes de apoyo y en las redes sociales que ayudan a fomentar las habilidades y la comprensión sobre la tecnología; desigualdad de contextos y de tiempo disponible como para experimentar y practicar con nuevos recursos tecnológicos y así sucesivamente. El aspecto más perjudicial de este tipo de división es que se está autoperpetuando e incluso acelerando; mientras más acceso a recursos ubicuos y oportunidades de aprendizaje se tiene, crecen las posibilidades de aumentar la distancia con aquellos que no lo tienen (p. 10).
}

Tomando como base todos estos motivos, toma especial relevancia la importancia de las políticas educativas generales relacionadas con la inclusión de las TIC y los MDD que se están llevando a cabo en la mayoría de las Comunidades Autónomas de nuestro país, materializándose en múltiples planes con el objetivo de paliar estas consecuencias. Debido a que la mera utilización de las TIC en el aula no se traduce en una 
mejora del proceso de enseñanza y aprendizaje, sino que requiere de un cambio metodológico y del rol del docente (Sanabria et al., 2017), se ha de invertir, necesariamente, en la formación de los docentes y en su empoderamiento, para que sean capaces de crear, experimentar y compartir los MDD con el resto de los miembros de la comunidad educativa. Para lo cual, se depende tanto de la conectividad de la que se disponga en los centros educativos como del uso que el docente haga de estos (Mur, 2016), a lo que se suma la formación, la escasez de recursos o la falta de tiempo como factores más influyentes en la implementación de las TIC (Cateriano-Chávez et al., 2021).

La introducción de los MDD en el proceso de aprendizaje, como ya hemos señalado con anterioridad, no sólo afecta al rol docente, sino que también genera cambios en el conjunto íntegro de la organización educativa y repercute directamente en las tareas desempeñadas por el alumnado y en el papel desempeñado por los materiales digitales, al formar parte de un sistema. Por ello, se transita de una estructura horizontal propia de la organización clásica, a una horizontal, expandida (Pardo et al., 2018).

Respecto al docente, acompaña, guía y diseña, actúa como facilitador, organizando recursos diversos, proporcionando andamiaje al proceso de enseñanza y aprendizaje, y promoviendo la reflexión. El estudiantado desempeña un papel proactivo, ha de desarrollar la capacidad de autogestión y motivación, quien también puede actuar como enseñante para sus compañeros y compañeras, desempeñando una labor de tutor. Y, dentro de este cambio de rol del profesorado, cabe remarcar su papel activo en la creación de MDD, incluso en los recursos proporcionados por los portales institucionales; no solamente es consumidor de los materiales ofrecidos por los distintos portales, sino que también forma parte de su creación, tanto de forma personal como colectiva (González et al., 2018).

En la etapa de Educación Infantil, en la que centramos esta investigación, es más complejo evaluar el efecto que las tecnologías están produciendo sobre el desarrollo del alumnado, debido a que es una etapa inicial en la que el proceso de aprendizaje se encuentra muy condicionado por el nivel madurativo de los niños y niñas. Al mismo tiempo, porque los fines con los que tradicionalmente se han empleado los contenidos digitales han sido fundamentalmente lúdicos o de apoyo al aprendizaje (Gabarda et al., 2021). Por tanto, aunque son sujetos que han crecido rodeados de dispositivos tecnológicos, a la hora de aplicar los MDD con una finalidad educativa es importante considerar sus habilidades motrices, sensoriales o cognitivas (Ramírez et al. (2021); tratar temáticas cercanas e interesantes que les motiven y capten su interés; y, sobre todo, gestionar la diversidad del aula para la que más que nunca se deberá aprovechar la flexibilidad y versatilidad que ofrecen los MDD (Huertas, 2021).

Para acabar, como conclusión de esta introducción, revelamos que, dado el gran potencial y protagonismo creciente que tienen los MDD en los centros educativos, este trabajo tiene como objetivo analizar en qué medida estos recursos digitalizados facilitan el tratamiento de la diversidad en el segundo ciclo de Educación Infantil (de 3 a 6 años) y permiten la realización en los centros de prácticas escolares mucho más inclusivas.

\section{MATERIAL Y MÉTODO}

Este trabajo se enmarca en el proyecto de investigación "Los materiales didácticos digitales en la Educación Infantil. Análisis y propuestas para su uso en la escuela y el hogar (Ref. RTI2018-093397-B-I00)”, del programa I+D+i orientado a los Retos de la sociedad (2018), del Ministerio de Ciencia, Innovación y Universidades; 
proyecto que, de manera resumida, tiene como objeto el análisis de las características técnicas y pedagógicas de diversos MDD del contexto español destinados al segundo ciclo de Educación Infantil.

Particularmente, en este artículo se ha tratado de dar un paso más del tratado en el análisis inicial y se realiza una exploración más profunda de los MDD. El objetivo específico abordado es conocer si la incursión de esta tipología de materiales didácticos puede favorecer una atención más personalizada e individualizada en la diversidad del alumnado de infantil, a través del desarrollo de prácticas educativas más accesibles, interactivas, motivadoras y dinámicas.

El desarrollo metodológico del proyecto en el que se encuadra este trabajo es mixto, puesto que se combinan estudios de naturaleza cualitativa y cuantitativa. No obstante, este texto se sitúa en el seno del primer estudio, en el que se ha empleado fundamentalmente el diseño cualitativo.

Concretamente, se ha seleccionado una muestra de 24 MDD dirigidos a Educación Infantil procedentes de plataformas o portales web de distinta naturaleza, institucional o comercial, y de aplicaciones informáticas gratuitas o de pago. Posteriormente, esta muestra se ha analizado mediante un instrumento previamente elaborado y validado en un proyecto de investigación anterior, disponible en Area (2020). Para concretar más, cabe destacar que no ha sido aplicado completamente todo el instrumento de análisis, este trabajo se ha focalizado en cuatro aspectos que guardan una relación directa con la atención a la diversidad y que aparecen detallados en la siguiente tabla.

TABLA 1. Aspectos para analizar la atención a la diversidad en los MDD

\begin{tabular}{|c|c|c|c|}
\hline $\begin{array}{l}\text { DISEÑO Y ACCESO } \\
\text { AL MDD }\end{array}$ & $\begin{array}{c}\text { RASGOS PEDAGÓGICOS } \\
\text { DEL MDD }\end{array}$ & $\begin{array}{l}\text { EVALUACIÓN } \\
\text { EN LOS MDD }\end{array}$ & $\begin{array}{l}\text { COMENTARIOS Y } \\
\text { VALORACIONES DEL } \\
\text { EVALUADOR }\end{array}$ \\
\hline $\begin{array}{l}\text { Diseño de la interfaz } \\
\text { (tipografía de letra, } \\
\text { uso de elementos } \\
\text { visuales o auditivos, } \\
\text { interactividad...). }\end{array}$ & $\begin{array}{l}\text { Finalidad, objetivos } \\
\text { didácticos, modelo } \\
\text { pedagógico, tipos de } \\
\text { aprendizaje, refleja distintos } \\
\text { ritmos de aprendizaje, etc. }\end{array}$ & $\begin{array}{l}\text { Realiza adaptaciones didácticas, } \\
\text { detalla criterios y estrategias de } \\
\text { evaluación o propone actividades de } \\
\text { evaluación grupales o individuales }\end{array}$ & $\begin{array}{c}\text { Fortalezas, debilidades y } \\
\text { aspectos a destacar. }\end{array}$ \\
\hline
\end{tabular}

Finalmente, para el análisis de la información se ha elaborado tres matrices de datos diferentes en función del origen de los distintos MDD: plataformas institucionales, plataformas comerciales o aplicaciones. Estas rejillas se han realizado con el propósito de facilitar el agrupamiento e interrelación de una gran cantidad de información de una manera muy visual (López et al., 2021).

\section{RESULTADOS}

Los MDD, de forma general, destacan por presentar un diseño bastante atractivo y motivador para el alumnado y por estructurar la información de forma clara y ordenada, lo que facilita su uso. Teniendo en cuenta las características evolutivas de los niños y niñas de infantil, los aspectos que contribuyen a mejorar la comprensión y captar la atención del alumnado son la utilización de interfaces simples, sin distractores y con fondos neutros o blancos; una tipografía de letra sencilla, legible, de colores diversos; y el apoyo de elementos visuales como ilustraciones, gráficos, dibujos o imágenes reales (véase la Figura 1). 
De forma más concreta, se han analizado MDD que incluyen componentes más específicos para gestionar la diversidad, tales como subtítulos del material auditivo y posibilidades de avanzar o retroceder de pantalla en cualquier momento, como sucede en el MDD "The secret forest: autumn" (http://links.uv.es/Wlx5sIV), o también la utilización de etiquetas visuales y de audio que favorecen el proceso de enseñanza a través de diferentes canales de información. Como ocurre, por ejemplo, en “L’ Oroneta i el rei” (https://links.uv.es/7Flsehs) y también en "Movemos el ratón” (http://links.uv.es/k4xpPd0).

Figura 1. Clasificación de actividades por grupos de edad integradas en la plataforma Educlan

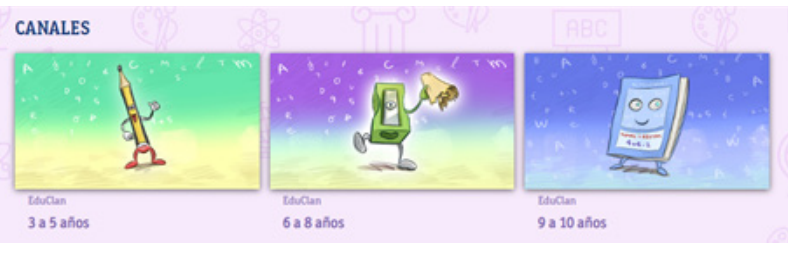

Figura 2. Pantalla de inicio del Proyecto Quiero (Globalizado) elaborado por Voramar (Santillana Educación)

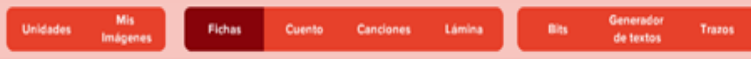

Unikad t Yo soy asi

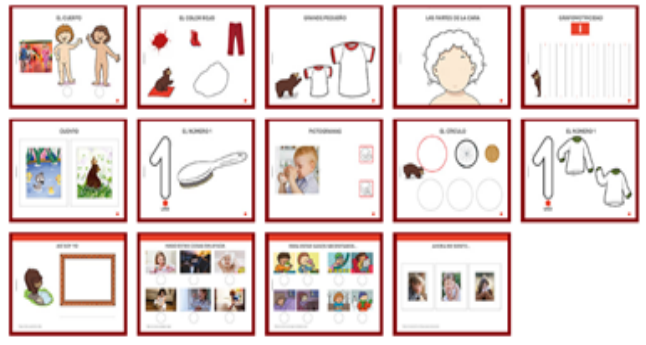

En la mayoría de los MDD analizados, se respetan y atienden los distintos ritmos de aprendizajes que coexisten en Educación Infantil, una muestra de esto son los recursos educativos "Bichos" (http://links.uv.es/d3utLGm), "Letras de colores" (http://links.uv.es/3nLmXQt) o “Miguel Hernández" (http://links.uv.es/BrOA8z8). Esta particularidad se consigue ofertando, desde las plataformas o portales web, una gran variedad de actividades graduales en las que se indica de manera general el nivel de dificultad, el curso o la edad del alumnado al que va dirigido. Un ejemplo de ello se puede comprobar en la Figura 2, en la que se puede observar que los materiales se dividen por grupos de edad bastante amplios. No obstante, son pocos los recursos que se complementan con un documento guía en el que se especifiquen los contenidos, objetivos, criterios de evaluación, etc., derivados o basados en los currículos oficiales y que brinden la posibilidad de realizar adaptaciones curriculares de las prácticas a realizar a los distintos ritmos de aprendizaje del alumnado. Este as-

pecto es de gran valor didáctico para una aplicación adecuada del recurso y aparece con mayor frecuencia en las plataformas desarrolladas por editoriales como Voramar, Santillana Educación o Grupo Edebé.

Si nos referimos al modelo pedagógico, se observa que predomina el aprendizaje por recepción guiada, a través de una estrategia metodológica expositiva con el objetivo de conseguir la comprensión y memorización de conceptos y enfocada a alcanzar un aprendizaje significativo y funcional. Por tanto, se observa que no se potencia la colaboración o el trabajo cooperativo ni la interacción del alumnado, y continúa imperando un modelo de enseñanza tradicional, en el que es necesaria la supervisión del adulto y que no beneficia el tratamiento de la diversidad ya que se trabaja de manera unidireccional sin tener en cuenta las individualidades del alumnado. Asimismo, la mayoría de los materiales son cerrados y estáticos y no ofrecen prácticamente posibilidades de edición o modificación. Sin embargo, es necesario subrayar la importancia del docente como sujeto mediador entre los materiales y el proceso de enseñanza, que cuenta con la capacidad para emplearlos con un enfoque más activo y contextualizado en las tareas y/o proyectos dependiendo de sus necesidades u objetivos previstos. 
Figura 3. Ejemplo de actividad con feedback auditivo y visual, tomada de Los increíbles MUN 5 y diseñada por ITBook y Santillana

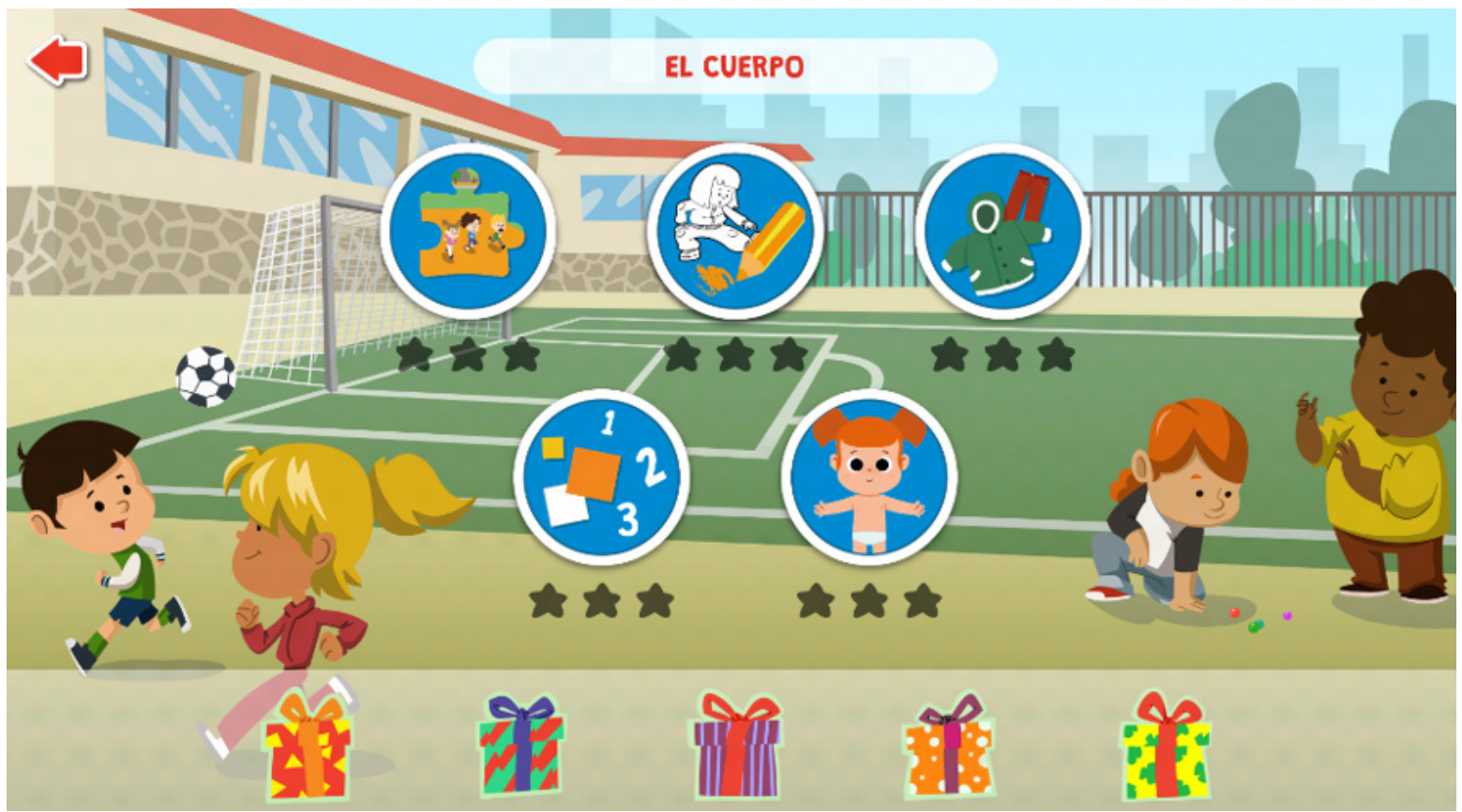

Teniendo en cuenta la edad de los usuarios, en casi todos los MDD de la muestra se combina la finalidad educativa con la lúdica, puesto que son recursos de aprendizaje caracterizados, en la mayoría de las ocasiones, por el juego que se considera una herramienta fundamental en el desarrollo integral de los niños y niñas de entre 3 y 6 años. Y en el objeto de este trabajo, emplear un enfoque lúdico en el aprendizaje es necesario y enriquecedor en alumnado que presenta necesidades educativas especiales ya que consigue captar su interés y generar más motivación e implicación en las actividades.

En el tratamiento de la diversidad, una parte fundamental dentro del proceso de enseñanza y aprendizaje es la evaluación. A pesar de que en algún MDD se aboga por una evaluación continua y formativa, se planifican actividades que permitan una autoevaluación y se detallan instrucciones para el alumnado y para el profesorado, en gran parte de los MDD seleccionados no se detallan criterios ni estrategias de evaluación, y tampoco se mencionan la posibilidad de realizar adaptaciones curriculares o metodológicas. Lo que sí figura en la mayoría de ellos y que es característico de los recursos interactivos es la retroalimentación inmediata sonora o visual que da información a los participantes sobre la consecución positiva o negativa de las distintas actividades, y que les anima a continuar participando, especialmente a aquellos que presentan dificultades. Por ejemplo, en la Figura 3 el alumnado es consciente de sus logros a través de estímulos auditivos, pero también visuales, pues cada logro se recompensa con la iluminación de una estrella.

Finalmente, con el propósito de mejorar los MDD y hacerlos mucho más inclusivos para el alumnado con ritmos de aprendizaje diversos, cabe señalar que solo en un material, denominado "Miguel Hernández" (http://links.uv.es/BrOA8z8), se ofrece la posibilidad de que el profesorado valore la calidad del mismo y su puesta en práctica, mediante una parrilla de preguntas en las que se puede reflexionar sobre la idoneidad del material y permite realizar posibles modificaciones. 


\section{DISCUSIÓN}

El segundo ciclo de Educación Infantil, como bien se sabe, es una etapa fundamental para el desarrollo integral de los niños y las niñas. Por ello, comprender y atender las necesidades del alumnado con necesidades de apoyo educativo en este período educativo tan temprano es esencial para garantizar poder alcanzar altos grados de bienestar en el futuro.

En el proceso de aprendizaje, los materiales didácticos son todos aquellos medios o dispositivos diseñados con el propósito de facilitar experiencias educativas más significativas. De los resultados de este trabajo se extrae que los MDD se presentan como recursos que motivan y captan la atención de todo el alumnado (Vidal et al., 2019), debido a que presentan interfaces muy atractivas con colores llamativos e imágenes, y porque cuentan con características que les distinguen de otros recursos más tradicionales (libros de texto, enciclopedias, diccionarios, etc.) como la interactividad o la capacidad multimedia. Al mismo tiempo, son recursos que dan respuesta a las demandas de una generación de niños y jóvenes con mayores competencias tecnológicas (Marín et al., 2021), y que se sienten mucho más atraídos por la información en formato digital que por la impresa en papel.

En general, la mayoría de los MDD analizados no contemplan de manera especial la diversidad de los alumnos y alumnas de infantil, ya que no se ofrece la posibilidad de realizar adaptaciones sobre su formato o contenido y tampoco se proporciona información más específica sobre objetivos, contenidos o criterios de evaluación que orienten al profesorado respecto a su aplicación en las aulas ordinarias inclusivas. Este hallazgo concuerda con las conclusiones extraídas del trabajo de Cepeda et al. (2017), donde se apunta a que los MDD presentan un enfoque muy disciplinar, y con escasa información sobre la puesta en práctica de los mismos en el desarrollo curricular. Además, tampoco cuentan con posibilidades de autoevaluación, un componente esencial a la hora de generar una reflexión con más rigurosidad acerca de su diseño y aplicación y que permita realizar mejoras o adaptaciones en los recursos didácticos empleados.

Por otro lado, es necesario destacar que la potencialidad de los MDD no reside solamente en sus características tecnológicas o de diseño, sino que también es importante el uso pedagógico que se haga de ellos (Vidal, 2015). Por eso, como señalan Rodríguez y Rodríguez (2016), es importante desarrollar otro tipo de discurso en relación con su integración y adaptación en el aula, que respete los rasgos básicos del proceso de aprendizaje en Educación Infantil y que destaque por emplear técnicas singulares como son la imitación, la acción o el juego.

En este cometido ocupa un papel esencial el profesorado, ya que deben ser los agentes encargados de emplear los recursos tecnológicos con estrategias metodológicas activas, cooperativas, inclusivas y centradas en el alumnado y que realmente supongan una innovación en el aula (Vidal et al., 2019). Así lo recogen los resultados de este trabajo, que avalan que la mayoría de los MDD proponen un aprendizaje por recepción, aunque dejan abierta la posibilidad de ser empleados de distintas formas en función de las necesidades del alumnado y de la disposición o competencia del profesorado. Pues, citando a Esnaola et al. (2019), el conjunto de los métodos, las estrategias o las técnicas de enseñanza que contextualizan y dan una estructura a los recursos tecnológicos son los que verdaderamente definen una buena práctica educativa. 


\section{CONCLUSIONES}

La introducción de los MDD en la etapa de Educación Infantil responde a la necesidad de dar respuesta a un alumnado que desde edades muy tempranas se encuentra rodeado de tecnología. Son recursos que, fundamentalmente, aportan mejoras al proceso de aprendizaje porque motivan al alumnado y captan su interés debido a su naturaleza multimedia e interactiva (Huertas, 2021). Por lo demás, son materiales muy visuales que presentan un diseño muy atractivo para todos los niños y niñas de edades comprendidas entre tres y seis años, basado en el uso de colores llamativos, imágenes, avatares o estímulos auditivos.

Para la atención a la diversidad, desafortunadamente, todavía son recursos que no favorecen un tratamiento diferenciado en función del contexto social o cultural o de su diversidad funcional. Por un lado, porque son materiales poco flexibles, que no permiten la edición con el objeto de realizar adaptaciones de formato o contenido que se ajusten adecuadamente a las necesidades del alumnado sobre el que se va emplear. Asimismo, no se acompañan de una guía didáctica que proporcione información más detallada sobre su puesta en práctica y que oriente al profesorado sobre los objetivos didácticos, criterios de evaluación o contenidos que se desarrollan. Además, la mayoría de ellos promueve un aprendizaje por recepción guiada, a través de una estrategia metodológica expositiva, un modelo educativo unidireccional, como ya hemos señalado, que no contempla los distintos ritmos o estilos de aprendizaje que conviven en un aula de infantil y que mantiene los roles desempeñados tradicionalmente tanto por el profesorado como por el alumnado.

Por último, cabe destacar que realizar un uso más efectivo y contextualizado de los MDD dependerá, coincidiendo con Mur (2016), del diseño metodológico que elija y utilice el profesorado, puesto que, en función de la estrategia con la que se emplee, el trabajo puede vincularse con procesos de enseñanza y aprendizaje más inclusivos, vivenciales, significativos, cooperativos o centrados en el alumnado. Por tanto, se concluye que la implementación de los MDD con un enfoque más comprensivo con la diversidad exigirá en el futuro de un gran trabajo y esfuerzo por parte del equipo docente de la etapa de infantil.

\section{REFERENCIAS}

Aguilar, I., De la Vega, J., Lugo, O., \& Zarco, A. (2014). Análisis de criterios de evaluación para la calidad de los materiales didácticos digitales. Revista Iberoamericana de Ciencia, Tecnología y Sociedad, 25(9), 73-89.

Area, M. (2017). La metamorfosis digital del material didáctico tras el paréntesis Gutenberg. Revista Latinoamericana de Tecnología Educativa, 16(2), 13-28. https://doi.org/10.17398/1695$\underline{288 X .16 .2 .13}$

Area, M. (Dir.) (2020).Escuel@ Digit@l, Los materiales didácticos en la Red. Graó.

Area, M., \& Pessoa, T. (2012). De lo sólido a lo líquido: las nuevas alfabetizaciones ante los cambios culturales de la Web 2.0. Comunicar, 38(9), 13-20. https://doi.org/10.3916/C38-2012-02-01
Burbules, N. C. (2012). El aprendizaje ubicuo y el futuro de la enseñanza. Encounters on Education, 13, 3-14. http://dx.doi. org/10.14507/epaa.v22.1880

Cateriano-Chavez, T. J., Rodríguez-Ríos, M. L., Patiño-Abrego, E. L., Araujo-Castillo, R. L., \& Villalba-Condori, K.O. (2021). Competencias digitales, metodología y evaluación en formadores de docentes. Campus Virtuales, 10(1), 153-162.

Cepeda, O., Gallardo, I. M., \& Rodríguez, J. (2017). La evaluación de los materiales didácticos digitales. Revista Latinoamericana de Tecnología Educativa, 16(2), 79-96. http://dx.medra. $\underline{\operatorname{org} / 10.17398 / 1695-288 X .16 .2 .79}$

Durán, S. (2021). Tecnologías para la enseñanza y el aprendizaje del alumnado con Trastorno del Espectro Autista: una revi- 
sión sistemática. Innoeduca. International Journal of Technology and Educational Innovation, 7(1), 107-121. https://doi. org/10.24310/innoeduca.2021.v7i1.9771

EDULLAB, CRIE y STELLAE (2019). Guía para la producción y uso de Materiales didácticos Digitales. Recomendaciones de buenas prácticas para productores, profesorado y familias. https:// riull.ull.es/xmlui/handle/915/16086

Esnaola, G., Reis, M., \& Marín, D. (2019). Del portal al aula: interacciones de los materiales didácticos digitales. Campus Virtuales, 8(2), 141-156.

Fernández, A. M., Domínguez, E., \& de Armas, I. (2011) Diez criterios para mejorar la calidad de los materiales didácticos digitales. En A. Sanz, J.A. López, \& A. Baratas (Coords.), VII Jornada Campus Virtual UCM: valorar, validar y difundir Campus Virtual (pp. 25-34). Universidad Complutense de Madrid,

Fombona, J., \& Vázquez-Cano, E. (2017). Posibilidades de utilización de la Geolocalización y Realidad Aumentada en el ámbito educativo. Educación XX1, 20(2), 319-342. https://doi. org/10.5944/educXX1.19046

Gabarda, V., Marín, D., \& Romero, M. M. (2021). Evaluación de recursos digitales para población infantil. EDMETIC, Revista de Educación Mediática y TIC, 10(1), 135-153. https://doi. org/10.21071/edmetic.v10i1.13125

González, C. J., Martín, S., \& Vega, A. (2018). Portales educativos: la producción de materiales didácticos digitales. @tic Revista d'Innovació Educativa, 20, 89-97.

Huertas, P. (2021). La digitalización educativa en educación infantil. eco. Revista Digital de Educación y Formación del profesorado, (18), 1-18.

López, S., Martín, S., \& Vidal, M.I. (2021). Análisis de aplicaciones móviles dirigidas a la infancia: características técnicas, pedagógicas, de diseño y contenido. Revista Iberoamericana de Educación, 85(1), 81-100. https://doi.org/10.35362/ rie8514013

Marín, D., Santana, P. J., \& Castro, M. M. (2021). Escuela Digital: estrategias y materiales didácticos digitales en Educación Infantil y Primaria. Revista Iberoamericana de Educación, 85(1), 9-13. https://doi.org/10.35362/rie4179-
Mur, L. (2016). La nueva brecha digital. El futuro de las nuevas tecnologías en Primaria desde la formación del Profesorado. REIFOP, 19(2), 301-313. https://doi.org/10.6018/reifop.19.2.189561

Page, D. (2016). The surveillance of teachers and the simulation of teaching. Journal of Education Policy, 32(1), 1-13. https://doi. org/10.1080/00313831.2018.1434825

Pardo, I., Waliño-Guerrero, M. J., \& San Martín, A. (2018). La “uberización" de los centros escolares: reestructuración del trabajo pedagógico mediante las plataformas digitales de contenido. Educatio Siglo XXI, 36(2), 187-208.

Peirats, J., Gallardo, I. M., San Martín, A., \& Cortés, S. (2015). Los contenidos curriculares digitalizados: Voces y silencios en el ámbito editorial. Educatio Siglo XXI, 33(3), 39-62. http:// dx.doi.org/10.6018/j/240801

Peirats, J., Gallardo I. M., San Martín, A., Cortés, S., López, M., Martí, T., Romero, M., \& Waliño, M. J. (2016). Protocolo para la evaluación y elección de materiales curriculares digitalizados. http://hdl.handle.net/10550/53577

Porter, J. (2018). Entering Aladdin's cave: Developing an app for children with Down syndrome. Journal of Computer Assisted Learning, 34(4), 429-439. https://doi.org/10.1111/jcal.12246

Ramírez, E., Cañedo, I., Orgaz, B., \& Martín. J. (2021). Evaluar competencias digitales en Educación Infantil desde las prácticas de aula. Pixel-Bit. Revista de Medios y Educación, (61), 37-69. https://doi.org/10.12795/pixelbit.85580

Rodríguez, J., Bruillard, E., \& Horsley, M. (Eds.) (2015). Digital Textbooks, What's New? IARTEM Servizo de Publicacións USC.

Rodríguez, J., \& Rodríguez, N. (2016). Revisión de la investigación publicada sobre el libro de texto digital en revistas, publicaciones y congresos internacionales de referencia. Profesorado. Revista de currículum y formación del profesorado, 20(1), 9-31.

San Martín, A., \& Peirats, J., (2018). Controversias en la transición del libro de texto en papel y electrónico a los contenidos digitales. RED. Revista de Educación a Distancia, 56(5), 1-17. http://dx.doi.org/10.6018/red/56/5

Sanabria, A. L., Álvarez, Q., \& Peirats, J. (2017). Las políticas educativas en la producción y la distribución de materiales didácti- 
Innoeduca. International Journal of Technology and Educational Innovation

López Marí, M., Sánchez Cruz, M., Peirats Chacón, J.

cos digitales. Revista Latinoamericana de Tecnología Educativa, 16(2), 63-77. https://doi.org/10.17398/1695-288X.16.2.63

Santana, P. J., Eirín, R., \& Marín, D. (2017). Análisis y evaluación de portales institucionales en España. Los casos de Canarias, Galicia y Valencia. Revista Latinoamericana de Tecnología Educativa, 16(2), 29-48. https://doi.org/10.17398/1695288X.16.2.29

Troseth, G. L., Russo, C. E., \& Strouse, G. A. (2016). What's next for research on young children's interactive media? Journal of Children and Media, 10(1), 54-62. https://doi.org/10.1080/17 $\underline{482798.2015 .1123166}$

Vidal, M. I., Vega, A., \& López, S. (2019). Uso de materiales didácticos digitales en las aulas de Primaria. Campus Virtuales, 8(2), 103-119. http://uajournals.com/ojs/index.php/campusvirtuales/article/view/516

Vidal, M. P. (2015). Medios, Materiales y Recursos Tecnológicos en la Educación Infantil. RELAdEI. Revista Latinoamericana de Educación Infantil, 4(1), 161-188. 